\title{
Versão Global do Princípio de Invariância Fuzzy, via extensão de Zadeh
}

\author{
Wendhel Raffa Coimbra ${ }^{1}$ \\ Departamento de Ciências Exatas, UFPB, Rio Tinto, PB \\ Luís Fernando Costa Alberto ${ }^{2}$ \\ Departamento de Engenharia Elétrica e de Computação, EESC-USP, São Carlos, SP
}

Resumo. Neste trabalho, apresentamos uma versão do princípio de invariância para uma classe de sistemas dinâmicos fuzzy que são obtidas a partir de sistemas dinâmicos determinísticos, via princípio da extensão de Zadeh, considerando apenas incertezas na condição inicial de um problema de valor inicial. A classe de sistemas dinâmicos considerada neste artigo são aqueles modelados por equações diferenciais autônomas. Este resultado é uma extensão do princípio de invariância clássico para equações diferenciais ordinárias para a classe de sistemas dinâmicos fuzzy. Esta versão do princípio de invariância fuzzy é útil para estudar o comportamento assintótico de soluções e obter estimativas de atratores e regiões de atração de sistemas dinâmicos fuzzy.

Palavras-chave. Equações Diferenciais, Sistemas Dinâmicos, Lyapunov, Princípio de Invariância, Sistemas Fuzzy

\section{Introdução}

A teoria de sistemas dinâmicos fuzzy aparece em diversas aplicações em engenharia $[2,3,7]$. Existem várias interpretações de sistemas dinâmicos fuzzy que estão relacionadas com as diferentes formas de diferenciabilidade no contexto fuzzy, tais como a $H$-diferenciabilidade adotada por Kaleva em 1987 [5] e a derivada de Hukuhara [9]. Oberguggenberger e Pittschamnn em 1999 determinam as soluções fuzzy aplicando a extenção de Zadeh sobre as soluções determinísticas de sistemas dinâmicos [8]. Para as equações diferenciais autônomas $\dot{x}=f(x)$, as propriedades das soluções fuzzy, via extensão de Zadeh, considerando a condição inicial como sendo um conjunto fuzzy, foram apresentadas em 2004 por Mizukoshi [7]. Estas soluções satisfazem as propriedades de um semigrupo e então as soluções podem ser caracterizadas como um fluxo sobre o conjunto formado pelos subconjuntos fuzzy do espaço de estados.

O Princípio de Invariância de LaSalle é uma das ferramentas mais importantes para estudar o comportamento assintótico de soluções de equações diferenciais sem a necessidade de conhecer explicitamente as soluções das equações diferenciais. Para isto, uma função escalar auxiliar, muitas vezes denominada função de Lyapunov, é utilizada. Neste

\footnotetext{
${ }^{1}$ wendhel@dcx.ufpb.br

${ }^{2}$ lfcalberto@usp.br
} 
artigo, uma versão do Princípio de Invariância Fuzzy via exntensão de Zadeh será desenvolvida para uma classe de sistemas dinâmicos Fuzzy com incertezas na condição inicial do Problema de Valor Inicial Fuzzy (PVIF). Além disso, aplicações deste resultado na obtenção de estimavas de atratores e regiões de atração de sistemas dinâmicos fuzzy são desenvolvidas.

\section{Preliminares}

Apresentaremos nesta seção conceitos fundamentais sobre a teoria fuzzy e sistemas dinâmicos fuzzy para melhor compreensão na Seção 3.

Considere $\mathcal{P}(X)$ o conjunto formado pelos subconjuntos de um conjunto $X$. Para cada $A \in \mathcal{P}(X)$ definimos a função característica $\chi_{A}: X \rightarrow\{0,1\}$ de $A$ por $\chi_{A}(x)=1$ se $x \in A$ ou -1 se $x \notin A$. Cada subconjunto fuzzy $F$ de $X$ é caracterizado por uma função de pertinência $\mu_{F}: X \rightarrow[0,1]$, que associa para cada $x \in X$ o grau de pertinência $\mu_{F}(x)$ de $x$ em $F$. A função de pertinência é uma generalização da função característica. Sendo $X$ um conjunto não vazio. Um subconjunto fuzzy $F$ de $X$ é um subconjunto $\left\{\left(x, \mu_{F}(x)\right): x \in X\right\}$ de $X \times[0,1]$ para alguma função $\mu_{F}: X \rightarrow[0,1]$.

O conjunto formado por todos os subconjuntos fuzzy de $X$ será denotado por $\mathcal{F}(X)$.

Se $A \in \mathcal{P}(X)$, isto é, $A \subset X$, então $\left\{\left(x, \chi_{A}(x)\right): x \in X\right\}$ é um subconjunto não vazio de $X \times[0,1]$. O subconjunto $A$ determina um subconjunto fuzzy de $X$ que será identificado aqui, com abuso de notação, por $\chi_{A}$. Para uma melhor distinção, o subconjunto fuzzy $\chi_{A}$ será denominado aqui, como na língua inglesa, um subconjunto crisp de $X$ (que se identifica como conjunto usual, no sentido clássico).

Sendo um subconjunto fuzzy $A$ em $F(X)$ definimos, para cada $\alpha \in(0,1]$, o conjunto $[A]^{\alpha} \subset X$ como sendo o conjunto dos elementos de $X$ tal que o grau de pertinência em $A$ é maior ou igual a $\alpha$. O conjunto $[A]^{\alpha} \subset X$ é denominado $\alpha$-nível de $A$ e é definido por $[A]^{\alpha}=\left\{x \in X: \mu_{A}(x) \geq \alpha\right\}$ para $\alpha \in(0,1]$. Além disso, O 0-nível de um subconjunto fuzzy $A$ é definido por $[A]^{0}=\overline{\bigcup_{\alpha \in(0,1]}[A]^{\alpha}}=\overline{\operatorname{supp}(A)}$ onde $\operatorname{supp}(A)=\left\{x \in X: \mu_{A}(x)>0\right\}$ é o suporte do subconjunto fuzzy $A$.

O princípio da extensão de Zadeh se baseia em estender o domínio de uma aplicação $f: X \rightarrow Y$ para os subconjuntos fuzzy em $\mathcal{F}(X)$. Observe que para cada $A \subset X$, temos que $f(A) \subset Y$. Agora, sendo $A \in \mathcal{F}(X)$, ou seja, $A$ é um subconjunto fuzzy $X$, então a maneira como a imagem se caracteriza é feita através do Princípio da Extensão de Zadeh, proposta em 1965, em que considera $f: X \rightarrow Y$ uma aplicação e $A$ um subconjunto fuzzy de $X$. A extensão de Zadeh $\hat{f}: \mathcal{F}(X) \rightarrow \mathcal{F}(Y)$ é a aplicação cuja imagem $\hat{f}(A)$ tem a função de pertinência $\mu_{\hat{f}(A)}(z)=\sup _{x \in f^{-1}(z)} \mu_{A}(x)$ se $f^{-1}(z) \neq \varnothing$ ou 0 se $f^{-1}(z)=\varnothing$, onde $f^{-1}(z)$ é a pré-imagem de $z$ (não necessariamente a inversa).

\section{$2.1 \quad$ O Subespaço $\mathcal{E}(X)$}

Adiante, iremos restringir a análise aos subconjuntos fuzzy de um conjunto $X$ cujos $\alpha$-níveis são subconjuntos compactos e não vazios em $X$, isto é, $\mathcal{E}(X):=\{A \in \mathcal{F}(X)$ : 
$[A]^{\alpha}$ é compacto e não vazio, $\left.\forall \alpha \in[0,1]\right\}$.

Os subconjuntos fuzzy que estão em $\mathcal{E}(X)$ serão denotados por letras minúsculas em negrito para diferenciar dos elementos de $X$. Os subconjuntos, no sentido clássico, do conjunto $\mathcal{E}(X)$ serão representados aqui por letras maiúsculas em negrito.

A seguir, iremos definir uma métrica sobre $\mathcal{E}(X)$ por meio da métrica de Hausdorff para subconjuntos compactos de $X$. Seja então $\mathcal{K}(X)$ o conjunto formado pelos subconjuntos compactos não vazios do espaço métrico $(X, d)$. Dados os conjuntos $A$ e $B$ em $\mathcal{K}(X)$, a distância entre os conjuntos é dada por: $\operatorname{dist}(A, B)=\sup _{a \in A} \inf _{b \in B} d(a, b)$ [6]. Sendo os conjuntos $A, B \in \mathcal{K}(X)$. A distância de Hausdorff entre $A$ e $B$ é definida por $d_{H}(A, B)=$ $\max \left\{\sup _{a \in A} \inf _{b \in B} d(a, b), \sup _{b \in B} \inf _{a \in A} d(a, b)\right\}=\max \{\operatorname{dist}(A, B), \operatorname{dist}(B, A)\}$.

Observe que $\left(\mathcal{K}(X), d_{H}\right)$ é um espaço métrico. Se $(X, d)$ é um espaço métrico completo então $\left(\mathcal{K}(X), d_{H}\right)$ também é um espaço métrico completo [1]. A partir da definição de Hausdorff, definimos a métrica para o conjunto $\mathcal{E}(X)$, ou seja, dados $\boldsymbol{u}, \boldsymbol{v} \in \mathcal{E}(X)$, dizemos que a distância, definida por $d_{\infty}$, entre $\boldsymbol{u}$ e $\boldsymbol{v}$, é dada por $d_{\infty}(\boldsymbol{u}, \boldsymbol{v})=\sup _{\alpha \in[0,1]} d_{H}\left([\boldsymbol{u}]^{\alpha},[\boldsymbol{v}]^{\alpha}\right)$. Agora, sendo os conjuntos $\boldsymbol{A}, \boldsymbol{B} \subset \mathcal{E}(U)$. A distância entre $\boldsymbol{A}$ e $\boldsymbol{B}$ é definida por $\operatorname{dist}(\boldsymbol{A}, \boldsymbol{B})=\sup _{\boldsymbol{x} \in \boldsymbol{A}} \inf _{\boldsymbol{y} \in \boldsymbol{B}} d_{\infty}(\boldsymbol{x}, \boldsymbol{y})$. Também podemos dizer que o conjunto $\boldsymbol{\omega}$ é limitado se, para quaisquer $\boldsymbol{x}, \boldsymbol{y} \in \boldsymbol{\omega}$, existe $r>0$ tal que a distância $d_{\infty}(\boldsymbol{x}, \boldsymbol{y}) \leq r$.

Proposição 2.1. [3] Suponha que $f: A \subset X \rightarrow Y$ seja contínua. Se $\boldsymbol{u} \in \mathcal{E}(X)$ é tal que $[\boldsymbol{u}]^{\alpha} \subset A, \forall \alpha \in[0,1]$, então $[\hat{f}(\boldsymbol{u})]^{\alpha}=f\left([\boldsymbol{u}]^{\alpha}\right)$ para todo $\alpha \in[0,1]$.

O princípio da extensão de Zadeh preserva algumas propriedades importantes, sendo que a principal é a continuidade [3].

\subsection{Problema de Valor Inicial Fuzzy via Extensão de Zadeh}

Neste trabalho, denominaremos por sistema dinâmico fuzzy, ou fluxo fuzzy, os sistemas dinâmicos definidos sobre o espaço $\mathcal{E}(X)$, obtidos pelo princípio da extensão de Zadeh de soluções determinísticas de equações diferenciais autônomas. Mizukoshi (2004) apresenta diversas interpretações do problema de valor inicial e uma delas é via princípio de extensão de Zadeh, a qual iremos tratar aqui. Considere o problema de valor inicial determinístico autônomo $\dot{x}=f(x(t)) ; x(0)=x_{0}(1)$, onde $f: U \subset \mathbb{R}^{n} \rightarrow \mathbb{R}^{n}$ é uma função contínua e $x_{0} \in \mathbb{R}^{n}$. Iremos admitir neste trabalho que as soluções do sistema (1) existem para todo $t \in \mathbb{R}$. Agora, considerando incerteza na condição inicial do sistema (1), temos o Problema de Valor Inicial Fuzzy (PVIF) dado por $\dot{x}=f(x(t)) ; x(0)=\boldsymbol{x}_{0}$, onde $\boldsymbol{x}_{0} \in \mathcal{E}\left(\mathbb{R}^{n}\right)$.

Definição 2.1. Sejam $U \subset \mathbb{R}^{n}$ aberto e $x_{0} \in U$. Dizemos que $\hat{\varphi}_{t}: \mathcal{E}(U) \rightarrow \mathcal{E}(U), t \in \mathbb{R}$ é uma solução fuzzy para a Equação (2) quando $\hat{\varphi}_{t}\left(\chi_{\left\{x_{0}\right\}}\right)=\chi_{\left\{\varphi_{t}\left(x_{0}\right)\right\}}, \forall x_{0}$, onde $\varphi_{t}: U \rightarrow U$ é a solução da equação (1).

Observa-se que, a partir da Definição 2.1, a solução fuzzy coincide com a solução do sistema (1) quando o conjunto $\boldsymbol{x}_{0}$ é um conjunto "crisp". Partindo da condição em que o PVI possua soluções e que possa ser estendido, então para obter a solução do PVIF 
com condição inicial fuzzy, o princípio de extensão de Zadeh aplicado nessas soluções de problema determinístico mantém os resultados de existência e unicidade das soluções do problema de valor inicial original e então considere $\varphi_{t}: U \rightarrow U$ um fluxo determinístico do problema (1). Então a extensão de Zadeh de $\varphi_{t}, \hat{\varphi}_{t}: \mathcal{E}(U) \rightarrow \mathcal{E}(U), t \in \mathbb{R}$ satisfaz as seguintes condições: a) $\hat{\varphi}_{0}\left(\boldsymbol{x}_{0}\right)=\boldsymbol{x}_{0}$; b) $\hat{\varphi}_{t+s}\left(\boldsymbol{x}_{0}\right)=\hat{\varphi}_{t} \circ \hat{\varphi}_{s}\left(\boldsymbol{x}_{0}\right)$ para todo $\boldsymbol{x}_{0} \in \mathcal{E}(U)$ e $t, s \in \mathbb{R}$.

\section{3 Órbitas, Conjuntos Limites e Conjuntos Invariantes}

Os conceitos de órbita e conjunto limite definidos para fluxos fuzzy $\hat{\varphi}_{t}$ são análogos aos conceitos definidos para fluxos em espaços métricos.

Uma órbita fuzzy $\gamma\left(\boldsymbol{x}_{0}\right) \subset \mathcal{E}(U)$ de um estado inicial $\boldsymbol{x}_{0} \in \mathcal{E}(U)$ como sendo o subconjunto no espaço de fase $\mathcal{E}(U)$ definido por $\gamma\left(\boldsymbol{x}_{0}\right)=\bigcup_{t \in \mathbb{R}} \hat{\varphi}_{t}\left(\boldsymbol{x}_{0}\right)=\left\{\hat{\varphi}_{t}\left(\boldsymbol{x}_{0}\right) \in \mathcal{E}(U): t \in \mathbb{R}\right\}$.

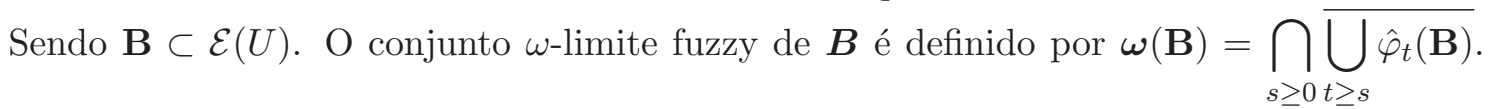
Por fim, $\omega(B), B \subset U$, o $\omega$-limite determinado pelo fluxo determinístico e consideremos o conjunto $\boldsymbol{\omega}(B) \subset \mathcal{E}(U)$ definido por $\boldsymbol{\omega}(B)=\left\{\boldsymbol{x} \in \mathcal{E}(U):[\boldsymbol{x}]^{0} \subset \omega(B)\right\}$. [3].

Temos algumas propriedades de $\boldsymbol{\omega}(B)$ e $\boldsymbol{\omega}(\boldsymbol{B})$ e suas relações, ou seja, sendo $\omega(A)$ e $A$ subconjuntos de $U$. O conjunto $\omega(A)$ atrai $A$ pelo fluxo determinístico $\varphi_{t}$ se, e somente se, $\boldsymbol{\omega}(A)$ atrai $\boldsymbol{A}=\left\{\boldsymbol{x} \in \mathcal{E}(U):[\boldsymbol{x}]^{0} \subset A \subset U\right\}$ pelo fluxo $\hat{\varphi}_{t}$ [3]. Assim, Como consequência temos que Se $\left[\hat{\varphi}_{t}\left(\boldsymbol{x}_{0}\right)\right]^{\alpha}, \forall \alpha \in[0,1]$ é limitada para $t \geq 0$ pelo fluxo fuzzy então $\boldsymbol{\omega}\left(\left[\boldsymbol{x}_{0}\right]^{0}\right)$ atrai $\boldsymbol{x}_{0}=\left\{\boldsymbol{x} \in \mathcal{E}(U):[\boldsymbol{x}]^{0} \subset\left[\boldsymbol{x}_{0}\right]^{0}\right\}$. Por fim, temos que se $\boldsymbol{\omega}(B)$ é limitado então $\omega(B)$ é limitado.

Em 1998, Román-Flores afirma que não podemos garantir que o conjunto $\boldsymbol{\omega}(B)$ seja um conjunto compacto [11]. Entretanto $\omega(B)$ é um conjunto compacto, então, o conjunto $\boldsymbol{\omega}(B)$ é fechado e limitado [3].

A partir do conjunto $B \subset U$, podemos definir o conjunto $\boldsymbol{B} \subset \mathcal{E}(U)$ da seguinte maneira: $\boldsymbol{B}:=\left\{\boldsymbol{x} \in \mathcal{E}(U):[\boldsymbol{x}]^{0} \subset B \subset U\right\}$. Com esta relação entre os conjuntos $B$ e $\boldsymbol{B}$, é interessante observar também a relação entre os conjuntos $\boldsymbol{\omega}(\boldsymbol{B})$ e $\boldsymbol{\omega}(B)$ em que o conjunto $\boldsymbol{\omega}(\boldsymbol{B})$ está contido em $\boldsymbol{\omega}(B)$, ou seja, $\boldsymbol{\omega}(\boldsymbol{B}) \subset \boldsymbol{\omega}(B)$ [10]. Não podemos afirmar nada a respeito da inclusão $\boldsymbol{\omega}(B) \subset \boldsymbol{\omega}(\boldsymbol{B})$.

Apresentamos o conceito de conjuntos invariantes para o fluxo fuzzy, em que um conjunto $\mathbf{S} \subset \mathcal{E}(U)$ é dito ser invariante (positivamente invariante) pelo fluxo $\hat{\varphi}_{t}$ se $\hat{\varphi}_{t}(\mathbf{S})=\mathbf{S}$ para todo $t \in \mathbb{R}(t \geq 0)$. Outros resultados podem ser encontrados em [10].

Há equivalências entre os conjuntos $S$ e $\boldsymbol{S}$, sendo $S \subset U$ um conjunto invariante pelo fluxo determinístico e considerando o conjunto $\mathbf{S} \subset \mathcal{E}(U)$ definido por $\mathbf{S}=\{\mathrm{x} \in \mathcal{E}(U)$ : $\left.[\boldsymbol{x}]^{0} \subset S\right\}$, então $S$ é invariante para $\varphi_{t}$ se, e somente se, $\mathbf{S}$ é invariante para o fluxo $\hat{\varphi}_{t}[3]$

O resultado anterior é fundamental, pois pela extensão de Zadeh de um fluxo determinístico, conseguimos obter conjuntos invariantes para o fluxo fuzzy. Temos a seguinte relação entre o $\omega$-limite $\omega(B)$ e $\boldsymbol{\omega}(B)$ : o conjunto $\omega(B), B \subset U$, é invariante por $\varphi_{t}$ se, e somente se, o conjunto $\boldsymbol{\omega}(B)$ é invariante pelo fluxo fuzzy $\hat{\varphi}_{t}$. [3] 


\section{Princípio de Invariância Fuzzy}

Uma versão do princípio de invariância para a classe de sistemas dinâmicos fuzzy é apresentada nesta seção. Este resultado é uma extensão do princípio de invariância clássico para essa classe de sistemas fuzzy [10].

Consideramos aqui um sistema com equações diferencais autônomas, onde temos incertezas apenas na condição inicial (PVIF via extensão de Zadeh). Apresentamos a seguir um princípio de invariância que é a versão global do resultado apresentado em [10].

Teorema 1 (Princípio de Invariância Fuzzy). Considere o sistema (2) e sejam $V: \mathbb{R}^{n} \rightarrow \mathbb{R}$ e $f: \mathbb{R}^{n} \rightarrow \mathbb{R}^{n}$ funções de classe $C^{1}$. Sejam $\hat{V}: \mathcal{E}\left(\mathbb{R}^{n}\right) \rightarrow \mathcal{E}(\mathbb{R})$ e $\hat{\dot{V}}: \mathcal{E}\left(\mathbb{R}^{n}\right) \rightarrow \mathcal{E}(\mathbb{R})$ as extensões de Zadeh de $V$ e $\dot{V}$, respectivamente. Admita que $[\hat{\dot{V}}(\boldsymbol{x})]^{\alpha} \subset(-\infty, 0], \forall \boldsymbol{x} \in$ $\mathcal{E}\left(\mathbb{R}^{n}\right), \forall \alpha \in[0,1]$ e defina $\boldsymbol{E}:=\left\{\boldsymbol{x} \in \mathcal{E}\left(\mathbb{R}^{n}\right): \hat{\dot{V}}(\boldsymbol{x})=\{0\}\right\}$. Seja $\boldsymbol{B}$ o maior conjunto invariante contido em $\boldsymbol{E}$. Então toda solução fuzzy $\hat{\varphi}_{t}$ limitada para $t \geq 0$ de $\dot{x}=f(x)$ converge para $\boldsymbol{B}$ quando $t \rightarrow \infty$.

Demonstração. Seja $\hat{\varphi}_{t}\left(\boldsymbol{x}_{0}\right)$ a solução fuzzy limitada para $t \geq 0$ e seja $\boldsymbol{\omega}\left(\left[\boldsymbol{x}_{0}\right]^{\alpha}\right)$ o conjunto $\boldsymbol{\omega}$-limite. Seja $y \in\left[\boldsymbol{x}_{0}\right]^{\alpha}$. Sabemos que $\omega(y) \subset \omega\left(\left[\boldsymbol{x}_{0}\right]^{\alpha}\right)$ é não vazio. Como $V$ é decrescente e $V\left(\varphi_{t}(y)\right)$ é limitada inferiormente, então $V\left(\varphi_{t}(y)\right) \rightarrow v(y) \in \mathbb{R}$ quando $t \rightarrow \infty$. Como $V\left(\varphi_{t}(y)\right)$ tende para $\omega(y)$ então da continuidade da $V$ temos que $V(x)=V(y), \forall x \in \omega(y)$. Da invariância de $\omega(y), \dot{V}(x)=0, \forall x \in \omega(y)$ e por $y$ ser arbitrário, então $\dot{V}(x)=0, \forall x \in$ $\omega\left(\left[\boldsymbol{x}_{0}\right]^{\alpha}\right)$. Tome $\boldsymbol{x} \in \boldsymbol{\omega}\left(\left[\boldsymbol{x}_{0}\right]^{\alpha}\right)$. Pela Definição ?? temos que $[\boldsymbol{x}]^{\alpha} \subset \omega\left(\left[\boldsymbol{x}_{0}\right]^{\alpha}\right), \forall \alpha \in[0,1]$ e pela Proposição 2.1 temos que $[\hat{\dot{V}}(\boldsymbol{x})]^{\alpha}=\dot{V}\left([\boldsymbol{x}]^{\alpha}\right), \forall x \in \boldsymbol{\omega}\left(\left[\boldsymbol{x}_{0}\right]^{\alpha}\right), \forall \alpha \in[0,1]$, então $\dot{V}\left([\boldsymbol{x}]^{\alpha}\right)=\{0\}$, pois $[\boldsymbol{x}]^{\alpha} \subset \omega\left(\left[\boldsymbol{x}_{0}\right]^{\alpha}\right)$. Logo $\boldsymbol{x} \in \boldsymbol{E}$ e $\boldsymbol{\omega}\left(\left[\boldsymbol{x}_{0}\right]^{\alpha}\right) \subset \boldsymbol{E}$. Portanto, podemos afirmar que $\boldsymbol{\omega}\left(\left[\boldsymbol{x}_{0}\right]^{\alpha}\right)$ atrai $\hat{\varphi}_{t}\left(\boldsymbol{x}_{0}\right)$, isto é, $\hat{\varphi}_{t}\left(\boldsymbol{x}_{0}\right) \rightarrow \boldsymbol{\omega}\left(\left[\boldsymbol{x}_{0}\right]^{\alpha}\right) \subset \boldsymbol{B}$ quando $t \rightarrow \infty$.

O exemplo a seguir ilustra o Teorema 1.

Exemplo 1. [4] Considere a equação diferencial de segunda ordem $\ddot{z}+2 a \dot{z}+z+z^{3}=0$, onde $a \in(0,1)$. O sistema pode ser descrito pela seguinte equação diferencial: $\dot{x}=y ; \dot{y}=$ $-x-2 a y-x^{3}(3)$.

Note que a origem $x_{e}=0$ é o único ponto de equilíbrio para $\varphi_{t}$. Então, a origem, $\boldsymbol{x}_{e}=\chi_{\{0\}}$ é um ponto de equilíbrio para $\hat{\varphi}_{t}$ [10]. Pode-se notar que para $a=0$ temos um sistema conservativo com a função energia: $V(x, y)=\frac{x^{2}+y^{2}}{2}+\frac{x^{4}}{4}$. Há uma perda de energia quando $a>0$ e portanto obtemos $\dot{V}$ dado por $\dot{V}(x, y)=-2 a y^{2} \leq 0, \forall x, y, \forall a>0$. Como $V(x, y) \rightarrow+\infty$ quando $\|x\|,\|y\| \rightarrow+\infty$ então $V$ é radialmente ilimitada e como $\dot{V}(x, y) \leq 0$ então $V$ é uma função decrescente. Assim, pela extensão de Zadeh, podemos dizer que $\hat{V}$ é radialmente ilimitada e que $\hat{\dot{V}}\left([\boldsymbol{x}]^{\alpha}\right) \subset(-\infty, 0], \forall \alpha \in(0,1]$ e portanto o conjunto $\boldsymbol{\Omega}_{L}$ é limitado para qualquer $L>0$. Definindo o conjunto $\boldsymbol{E}:=\left\{\boldsymbol{x} \in \mathcal{E}\left(\mathbb{R}^{n}\right)\right.$ : $\boldsymbol{x}=\{0\}\}$, temos que todas as hipóteses do Teorema estão satisfeitas e portanto as soluções tendem para o maior conjunto invariante $\boldsymbol{B}$ contido em $\boldsymbol{E}$. Como a origem é o maior conjunto invariante, então as soluções tendem para origem quando $t \rightarrow \infty$.

A Figura 1 apresenta a projeção da solução fuzzy $\hat{\varphi}_{t}\left(\boldsymbol{x}_{0}\right)$ no espaço de fase. 


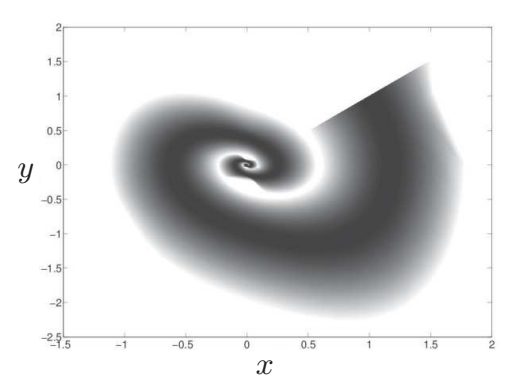

Figura 1: Estimativa do atrator global do sistema (3). A trajetória representa a evolução da projeção de $\left.\hat{\varphi}_{t}\left(\boldsymbol{x}_{0}\right)\right)$. As regiões mais escuras representam posições com maior valor de função de pertinência.

A Figura 2 apresenta a projeção da solução fuzzy $\hat{\varphi}_{t}\left(\boldsymbol{x}_{0}\right)$ para tempos diferentes $(t=0$ a $t=20)$.

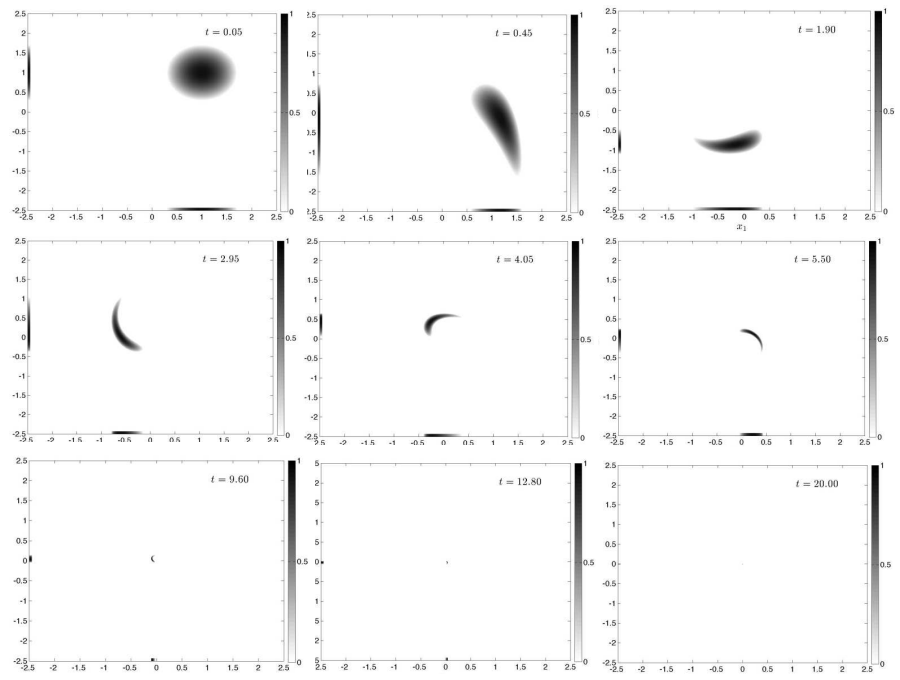

Figura 2: A trajetória representa a evolução da projeção de $\hat{\varphi}_{t}\left(\boldsymbol{x}_{0}\right)$ em tempos diferentes. Os tempos capturados são $t=0.05, t=0.45, t=1.90, t=2.95, t=4.05, t=5.50, t=9.60, t=12.80 \mathrm{e}$ $t=20.00$, onde a evolução tende para a origem.

\section{Conclusões}

Uma versão global do princípio de invariância para a classe de sistemas dinâmicos fuzzy foi provada neste artigo. O objetivo principal desta versão global do princípio de invariância fuzzy é estimar atratores e região de estabilidade para classe de sistemas dinâmicos fuzzy, com incertezas apenas na condição inicial de um PVIF. 


\section{Agradecimentos}

Agradecemos a Profa. Dra. Luciana Takata Gomes pela ajuda e ao CNPq pelo suporte financeiro parcial deste trabalho.

\section{Referências}

[1] C. D. Aliprantis and K. C. Border. Infinite Dimensional Analysis. Springer, New York, 2005. ISBN: 978-3-540-29586-0.

[2] L. C. Barros, Sobre Sistemas Dinâmicos Fuzzy: Teoria e Aplicações, Tese de Doutorado, Unicamp, 1997.

[3] M. S. Cecconello, Sistemas Dinâmicos em Espaços Métricos Fuzzy - Aplicações em Biomatemática, Tese de Doutorado, Unicamp, 2010.

[4] J.K. Hale and H. Kocak. Dynamics and Bifurcation. vol. 3 de Text in Applied Mathematics. Springer - Verlag, 1991. ISBN-10: 0387971416.

[5] O. Kaleva, Fuzzy differential equations, Fuzzy Sets and Systems, 1987. DOI: 10.1016/0165-0114(87)90029-7.

[6] E. Kreyszig. Introductory Functional Analysis With Applications. John Wiley \& Sons, 1978. ISBN-10: 0471504599.

[7] M. T. Mizukoshi, Estabilidade de Sistemas Dinâmicos Fuzzy, Tese de Doutorado, Unicamp, 2004.

[8] M. Oberguggenberger and S. Pittschmann, Differential equations with fuzzy parameters, Mathematical and Computer Modelling of Dynamical Systems, 1999. DOI: 10.1076/mcmd.5.3.181.3683.

[9] M. L. Puri and D. A. Ralescu, Differentials of fuzzy functions, J. Math. Analysis and Applications, 1983. DOI: 10.1016/0022-247X(83)90169-5.

[10] W. C. Raffa, Extensão do princípio de invariância de lasalle para sistemas periódicos e sistemas fuzzy, Tese de Doutorado, USP, 2016.

[11] H. Román-Flores, The of compactness $e(x)$, Appl. Math. Lett., 1998. DOI: 10.1016/S0893-9659(98)00003-2. 